EDITORIAL

\title{
Stress haemodynamics for asymptomatic mitral regurgitation: how much does it help?
}

\section{J-L Monin}

Heart 2005;91:1383-1384. doi: 10.1136/hrt.2004.059238

\section{Stress haemodynamics for asymptomatic mitral valve regurgitation may prove helpful in determining the optimal timing for surgery}

M itral regurgitation (MR) is the second most common valve disease in western countries after aortic stenosis, representing $32 \%$ of single native left sided valve disease in a recent European survey. ${ }^{1}$ The most frequent aetiology of MR is degenerative (valve prolapse or flail leaflets) for which valve repair is widely accepted as the optimal surgical treatment, considering a lower perioperative mortality ${ }^{2}$ and better long term outcome explained by better preservation of left ventricular (LV) function and avoidance of prosthetic valve related complications. ${ }^{3}$ Valve repair is obviously an incentive to early surgery in case of severe degenerative MR. However, the decision to operate on an asymptomatic patient remains a source of debate. ${ }^{45}$ In this setting, the detection of asymptomatic LV dysfunction is a major issue of concern. According to current practice guidelines, surgery is recommended in patients with severe MR in case of LV ejection fraction $<60 \%$ and/or LV end systolic diameter $>45 \mathrm{~mm}$ (or $26 \mathrm{~mm} / \mathrm{m}^{2}$ of body surface area). ${ }^{45}$ Unfortunately, the assessment of LV systolic function at rest can be misleading, due to a normal or near normal ejection fraction explained by the decreased afterload induced by severe MR. Therefore, new parameters are needed to assess LV systolic function in patients with asymptomatic MR.

\section{EVALUATION OF LV FUNCTION}

In this issue of Heart, Lee et al, ${ }^{6}$ provide new insight into the dynamic evaluation of LV systolic function and its prognostic implications. This study follows a previous report by Leung et $a l^{7}$ which demonstrated that postoperative LV dysfunction after mitral valve repair was best predicted by the dynamic evaluation of $\mathrm{LV}$ function (during exercise) than by rest indexes. In the present study, LV ejection fraction was measured at rest and immediately after exercise; LV contractile reserve (CR), was defined by a $4 \%$ increase in LV ejection fraction after exercise. ${ }^{6}$ The results show that $\mathrm{CR}$ was present in approximately two thirds of patients $(\mathrm{CR}+)$ and absent in the remaining third $(\mathrm{CR}-)$. Valve surgery was performed (at the discretion of the referring physicians, aware of all investigation results) in $39 \%$ of CR+ patients and in $84 \%$ of $\mathrm{CR}-$ patients, with the same proportion of valve
Lattre de Tassigny, 94010 Créteil, France; jeanluc. monin@free.fr repair in both groups. In the group as a whole, as well as in the surgical group, independent predictors of postoperative LV function were CR and rest LV end systolic volumes. Importantly, the evaluation of CR had an incremental value over rest end systolic volumes to predict follow up ejection fraction. Furthermore, patients with impaired CR had a worse clinical outcome whatever the treatment applied: higher rate of cardiac events under medical treatment or postoperatively; all five late postoperative cardiac events (heart failure, $\mathrm{n}=3$, or atrial fibrillation, $n=2$ ) occurred in the group of patients with exhausted CR. In contrast, an intact CR predicted a favourable outcome for both surgically or medically treated patients.

Prior studies evaluating LV CR in asymptomatic valve regurgitation are scarce. Wahi et $a l^{8}$ evaluated LV ejection fraction at rest and postexercise in 61 patients with asymptomatic aortic regurgitation and without coronary artery disease, 26 of whom underwent aortic valve replacement. Their results showed that CR was superior to rest indexes to predict postoperative LV function as well as the development of LV dysfunction under medical treatment. ${ }^{8}$ Thus, the results of Lee et $a l^{6}$ supported by Wahi et $a l^{8}$ demonstrate that LV CR is of prognostic importance in patients with asymptomatic or minimally symptomatic left sided valve regurgitation.

\section{STUDY LIMITATION}

One limitation of this study is that when CR is impaired, postoperative outcome is already compromised, due to the presence of latent LV dysfunction. From these results, there is no doubt that all patients with exhausted CR should undergo prompt surgery. But on the other hand, the fact that postoperative LV dysfunction or late cardiac events occur, respectively, in 19\% and $24 \%$ of patients with impaired CR might be an incentive to operate on the patients before the loss of CR. One possible explanation for the relatively high prevalence of postoperative LV dysfunction is the nearly one year time interval between initial testing and surgery in both groups. We do not know if the poor postoperative results in $\mathrm{CR}-$ patients were due to latent $\mathrm{LV}$ dysfunction at study entry or to the deterioration of LV function during the time elapsed while awaiting surgery. Another explanation may be that a $4 \%$ increase in LV ejection fraction during exercise is not enough to predict that postoperative LV dysfunction will not occur; thus

Abbreviations: $C R$, contractile reserve; $L V$, left ventricular; $M R$, mitral regurgitation 
future studies should aim at defining the best cut-off value in order to prevent postoperative LV dysfunction and cardiac events in most patients. Furthermore, as stated by the authors, the time interval before loss of CR is not known. Obviously, serial exercise testing in order to detect an eventual loss of CR is of interest for patients with intact CR who are treated medically. Unfortunately, despite repeat evaluation of CR in 35 patients, no data on serial testing were provided in this study.

In conclusion, this study and others ${ }^{7}{ }^{8}$ support the idea that stress haemodynamics for asymptomatic valve regurgitation is of prognostic importance and might be helpful in order to select the optimal timing for surgery. Concerning severe asymptomatic MR and according to current guidelines, surgery is indicated in case of latent LV dysfunction (LV ejection fraction $<60 \%$ and/or LV end systolic diameter $>45 \mathrm{~mm}$ ), atrial fibrillation or high systolic pulmonary pressure (>50 mm Hg at rest),${ }^{45}$ and stress haemodynamics have no indication in this case. Although it is more debatable, surgery can reasonably be considered for asymptomatic patients without LV dysfunction, in case of low operative risk (age $<70$ years, no co-morbidity), and a high likelihood of valve repair on the basis of functional echocardiographic analysis and the surgeon's experience. ${ }^{5}$ Conversely, stress haemodynamics may be helpful in asymptomatic severe MR when there is a doubt regarding the potential benefit of surgery: older patients, significant co-morbidities, doubt about the feasibility of valve repair. Further studies on large populations are needed to assess the best parameters and threshold values for stress haemodynamics in this setting.

\section{REFERENCES}

1 lung B, Baron G, Butchart EG, et al. A prospective survey of patients with valvular heart disease in Europe: the Euro heart survey on valvular heart disease. Eur Heart J 2003;24:1231-43.

2 Enriquez-Sarano M, Schaff HV, Orszulak TA, et al. Valve repair improves the outcome of surgery for mitral regurgitation. A multivariate analysis. Circulation 1995;91:1022-8.

3 Lee EM, Shapiro LM, Wells FC. Superiority of mitral valve repair in surgery for degenerative mitral regurgitation. Eur Heart J 1997; 18:655-63.

4 Bonow RO CB, de Leon AC, et al. ACC/AHA guidelines for the management of patients with valvular heart disease. A report of the American College of Cardiology/American Heart Association task force on practice guidelines (committee on management of patients with valvular heart disease). J Am Coll Cardiol 1998;32:1486-588

5 lung B, Gohlke-Bärwolf C, Tornos P, et al. Recommendations on the management of the asymptomatic patient with valvular heart disease. Eur Heart J 2002;23:1253-62.

6 Lee R, Haluska B, Leung DY, et al. Functional and prognostic implications of left ventricular contractile reserve in patients with asymptomatic severe mitral regurgitation. Heart 2005;91:1407-12.

7 Leung DY, Griffin BP, Stewart WJ, et al. Left ventricular function after valve repair for chronic mitral regurgitation: predictive value of preoperative assessment of contractile reserve by exercise echocardiography. J Am Coll Cardiol 1996;28:1198-205.

8 Wahi S, Haluska B, Pasquet A, et al. Exercise echocardiography predicts development of left ventricular dysfunction in medically and surgically treated patients with asymptomatic severe aortic regurgitation. Heart 2000;84:606-14.

\section{IMAGES IN CARDIOLOGY}

\section{Waving back: left atrial fibroelastoma mimicking an appendage thrombus}

doi: $10.1136 /$ hrt.2004.058784

A 63 year old woman with severe mitral regurgitation and permanent atrial fibrillation was referred for surgical replacement of the mitral valve Transoesophageal echocardiography (TOE) performed at another facility suggested that this patient had an obvious thrombus in the left atrial appendage (LAA). TOE was repeated to evaluate whether surgical repair of the mitral valve was possible. Bileaflet prolapse of the mitral valve caused by myxomatous degeneration was seen. The left ventricular size and ejection fraction were normal; the left atrium was enlarged. Notably, there was no spontaneous echo contrast in the left atrium; the LAA velocity was mildly decreased. Evaluation of the LAA indicated the presence of a curious structure resembling a sea anemone exhibiting a "hand waving motion" (panel A: arrow indicates the mass located in the left atrial appendage. LA, left atrium; LV, left ventricle). Finger-like projections distinguished the latter as

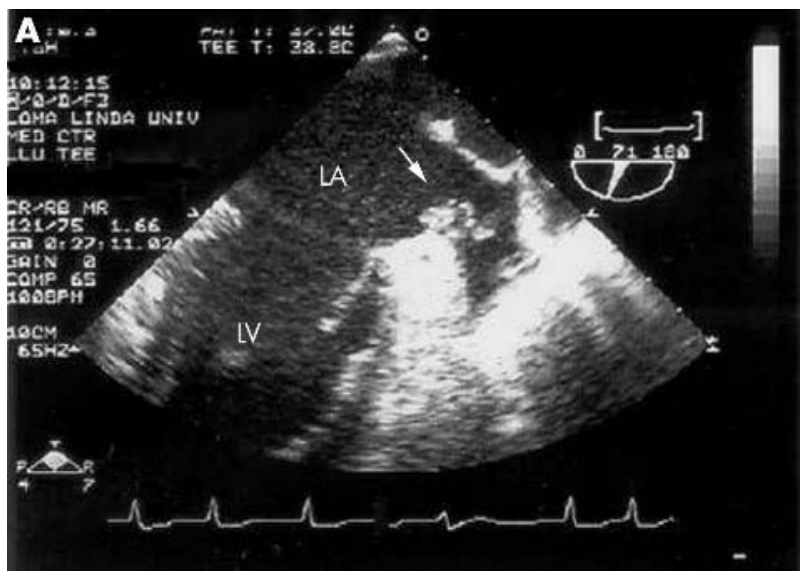

an atrial fibroelastoma. The LAA and fibroelastoma were resected during an uneventful surgery. Macroscopic examination and histological preparations confirmed the diagnosis; frond-like protrusions are clearly visible under microscopy (panel B). Although conventional wisdom would suggest that the structure in question was a thrombus, no echocardiographic data suggesting significant impairment of left atrial function were seen. Fibroelastomas occur primarily on valves, namely the mitral and aortic. They have been rarely described in the left atrial appendage, and may be confused with thrombi.

\section{Perzanowski R C Bansal N Wang chrisperz@att.net}

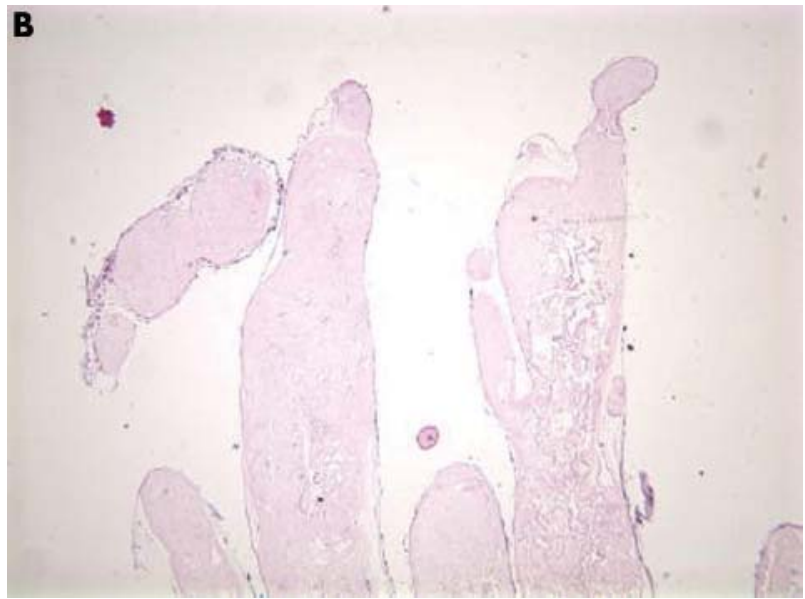

